\title{
Identification of Minimal Hepatic Encephalopathy in Patients with Cirrhosis Based on White Matter Imaging and Bayesian Data Mining
}

H.-J. Chen, R. Chen, M. Yang, G.-J. Teng, and E.H. Herskovits

\begin{abstract}
BACKGROUND AND PURPOSE: White matter abnormalities have been demonstrated to play an important role in minimal hepatic encephalopathy. In this study, we aimed to evaluate whether WM diffusion tensor imaging can be used to identify minimal hepatic encephalopathy among patients with cirrhosis.

MATERIALS AND METHODS: Our study included 65 patients with cirrhosis with covert hepatic encephalopathy (29 with minimal hepatic encephalopathy and 36 without hepatic encephalopathy). Participants underwent DTI, from which we generated mean diffusivity and fractional anisotropy maps. We used a Bayesian machine-learning technique, called Graphical-Model-based Multivariate Analysis, to determine WM regions that characterize group differences. To further test the clinical significance of these potential biomarkers, we performed Cox regression analysis to assess the potential of these WM regions in predicting survival.

RESULTS: In mean diffusivity or fractional anisotropy maps, 2 spatially distributed WM regions (predominantly located in the bilateral frontal lobes, corpus callosum, and parietal lobes) were consistently identified as differentiating minimal hepatic encephalopathy from no hepatic encephalopathy and yielded $75.4 \%-81.5 \%$ and $83.1 \%-92.3 \%$ classification accuracy, respectively. We were able to follow 55 of 65 patients (median = 18 months), and 15 of these patients eventually died of liver-related causes. Survival analysis indicated that mean diffusivity and fractional anisotropy values in WM regions were predictive of survival, in addition to the Child-Pugh score.
\end{abstract}

CONCLUSIONS: Our findings indicate that WM DTI can provide useful biomarkers differentiating minimal hepatic encephalopathy from no hepatic encephalopathy, which would be helpful for minimal hepatic encephalopathy detection and subsequent treatment.

ABBREVIATIONS: FA = fractional anisotropy; GAMMA = Graphical-Model-based Multivariate Analysis; $\mathrm{HE}=$ hepatic encephalopathy; $\mathrm{HR}=$ hazard ratio; $\mathrm{MD}=$ mean diffusivity; $\mathrm{MHE}=$ minimal hepatic encephalopathy; $\mathrm{NHE}=$ no hepatic encephalopathy

$\mathrm{H}$ epatic encephalopathy (HE) is a serious and frequent complication of cirrhosis, ${ }^{1}$ which can increase the risk of death. ${ }^{2}$ As the mildest form of $\mathrm{HE}$, minimal hepatic encephalopathy (MHE) is defined by impaired performance on psychometric or neu-

Received April 29, 2014; accepted after revision September 5.

From the Jiangsu Key Laboratory of Molecular and Functional Imaging (H.-J.C., M.Y., G.J.T.), Department of Radiology, Zhongda Hospital, Medical School, Southeast University, Nanjing, China; Department of Diagnostic Radiology and Nuclear Medicine (R.C., E.H.H.), University of Maryland School of Medicine, Baltimore, Maryland; and Department of Radiology (H.-J.C.), The First Affiliated Hospital of Nanjing Medical University, Nanjing, China.

This work was supported by the grants from the National Basic Research Program of China (973 Program: No. 2013CB733800, No. 2013CB733803), National Natural Science Foundation of China (No. 30910103905), Jiangsu Provincial Special Program of Medical Science (BL2013029), and Scientific Research Foundation of Graduate School of Southeast University (No. YBJJ1243).

Please address correspondence to Edward H. Herskovits, MD, PhD, Department of Diagnostic Radiology and Nuclear Medicine, University of Maryland School of Medicine, Baltimore, Maryland 21201; e-mail: ehh@ieee.org; or Gao-Jun Teng, MD, Jiangsu Key Laboratory of Molecular and Functional Imaging, Department of Radiology, Zhongda Hospital, Medical School, Southeast University, Nanjing 210009 , China; e-mail: giteng@vip.sina.com rophysiologic testing, despite normal mental status. ${ }^{1,3}$ MHE is associated with impaired quality of $\mathrm{life}^{4}$ and predicts conversion to overt $\mathrm{HE},{ }^{5}$ which can result in subsequent mortality. ${ }^{2}$ Meanwhile, MHE represents a treatable and reversible stage of $\mathrm{HE}^{6}$; it is, therefore, important to identify MHE to optimize treatment and prognosis.

Abnormalities in brain white matter, such as low-grade edema ${ }^{7-11}$ and structural impairments, ${ }^{12-14}$ have been well-revealed in MHE by MR imaging. These WM abnormalities are believed to be responsible for poor neurologic performances ${ }^{12,15,16}$ and brain dysfunctions ${ }^{17,18}$ in MHE and to be associated with HE development, ${ }^{9,11,16}$ suggesting that WM imaging may be useful in characterizing potential MHE biomarkers. Of WM imaging techniques, diffusion tensor imaging, measuring the appropriate metrics such as mean diffusivity (MD), reflecting water movement across cell membranes, and fractional anisotropy (FA), reflecting microstructural in-

\footnotetext{
- Indicates open access to non-subscribers at www.ajnr.org

Indicates article with supplemental on-line photo.

http://dx.doi.org/10.3174/ajnr.A4146
}

AJNR Am J Neuroradiol 36:481-87 Mar 2015 www.ajnr.org 
Table 1: Demographic and clinical characteristics of subjects

\begin{tabular}{lccc}
\multicolumn{1}{c}{ Characteristic } & $\begin{array}{c}\text { Patients with NHE } \\
(\boldsymbol{n}=\mathbf{3 6})\end{array}$ & $\begin{array}{c}\text { Patients with MHE } \\
(\boldsymbol{n}=\mathbf{2 9})\end{array}$ & $\boldsymbol{P}$ Value \\
\hline Age (yr) & $50.1 \pm 8.9$ & $52.8 \pm 8.4$ & .227 \\
Sex (male/female) & $34 / 2$ & $26 / 3$ & .471 ( $\chi^{2}$ test) \\
Education (yr) & $8.5 \pm 2.9$ & $7.6 \pm 2.3$ & .158 \\
Etiology of cirrhosis (HBV/alcoholism/ & $29 / 4 / 1 / 2$ & $22 / 0 / 4 / 3$ & - \\
HBV+alcoholism/other) & & & \\
Child-Pugh stage (A/B/C) & $19 / 12 / 5$ & $8 / 10 / 11$ & - \\
Child-Pugh score & $7.0 \pm 2.2$ & $8.4 \pm 2.2$ & .016 \\
Previous history of overt HE (yes/no) & $7 / 29$ & $10 / 19$ & .170 \\
Number Connection Test A (sec) & $44.5 \pm 10.8$ & $81.4 \pm 23.8$ & $<.001$ \\
Digit Symbol Test (raw score) & $40.2 \pm 8.7$ & $23.6 \pm 7.7$ & $<.001$ \\
Block Design Test (raw score) & $29.3 \pm 8.8$ & $17.7 \pm 6.5$ & $<.001$ \\
\hline
\end{tabular}

Note:- HBV indicates hepatitis B virus. tients were identified as having MHE if they had abnormal scores in any of these 3 neurocognitive tests. ${ }^{19,27}$

Exclusion criteria were the presence of overt $\mathrm{HE}$ at baseline, known presence of neuropsychiatric diseases, significant nonhepatic diseases (such as decompensated heart and respiratory or renal failure), and uncontrolled endocrine or metabolic diseases (such as diabetes mellitus and thyroid dysfunction). Subjects on psychoactive medications or with alcohol abuse 6 months before the study were also excluded. tegrity of WM, is demonstrated to reveal the neuropathologic processes of MHE through group-level comparison. ${ }^{8,9,12,14,15,17}$ To date, however, there is no study using DTI-derived feature maps to discriminate patients with and without MHE in cirrhosis at the individual level; this discrimination remains an important question for clinicians. ${ }^{6,19}$ The only related study is the report of Sugimoto et al, ${ }^{16}$ which used a DWI-based metric (apparent diffusion coefficient) instead of DTI to classify patients with or without MHE, but in which patients in the intermediate state were excluded; this method obviously results in inadequate assessment. Few studies to date have been conducted to detect MHE by using FA as a biomarker.

In this study, our goal was to investigate whether DTI feature maps can distinguish patients with cirrhosis with MHE from those without (NHE) by using a Bayesian voxel-based machine-learning technique called Graphical-Model-based Morphometric Analysis (GAMMA; http://www.nitrc.org/projects/ gamma_suite). ${ }^{20-22}$ GAMMA is a nonparametric, multivariate, Bayesian approach to modeling structure-function associations, which allows dimension reduction and predictive modeling in a unified framework and has been validated both on structural and functional MR imaging data ${ }^{21,23}$ and has been used in several studies related to neurologic diseases. ${ }^{22,24}$ To further validate identified biomarkers, we assessed their abilities to predict survival among patients with cirrhosis with covert hepatic encephalopathy, given the relationship between MHE and mortality: The presence of MHE has been observed to have a prognostic impact on mortality among patients with liver cirrhosis. ${ }^{25,26}$

\section{MATERIALS AND METHODS \\ Subjects}

This study was approved by the Research Ethics Committee of Affiliated Zhongda Hospital, Southeast University, China; 65 patients with clinically proved cirrhosis with covert HE (29 with MHE and 36 with NHE) were included after written informed consent was obtained (Table 1).

For psychometric assessment, a battery of neuropsychiatric tests, including the Number Connection Test A, Digit Symbol Test, and Block Design Test, were administered to all participants. These 3 neuropsychiatric tests are widely used in MHE detection, ${ }^{1,3,16}$ development of new diagnostic methods for MHE, ${ }^{19,27}$ and survival analysis of patients with cirrhosis. ${ }^{16,28} \mathrm{~Pa}-$

\section{MR Imaging Acquisition}

MR imaging was performed by using a $1.5 \mathrm{~T}$ scanner (Vantage Atlas; Toshiba Medical Systems, Tokyo, Japan). DTI sequences were acquired by using a 6-direction, single-shot, spin-echo EPI sequence, with $\mathrm{TR}=9450 \mathrm{~ms}, \mathrm{TE}=100 \mathrm{~ms}, \mathrm{FOV}=260 \times 260$ $\mathrm{mm}$, matrix $=128 \times 128$, section thickness/gap $=3.0 / 0 \mathrm{~mm}$, $b=1000 \mathrm{~s} / \mathrm{mm}^{2}$, and NEX $=3$. The $3 \mathrm{D}$ high-resolution $(1.0 \times$ $1.0 \times 1.5 \mathrm{~mm}$ ) T1-weighted images were also acquired for the registration of DTI.

\section{DTI Processing}

We used the fMRI of the Brain Software Library (FSL; http:// fsl.fmrib.ox.ac.uk/fsl) to process the DTI data. We corrected the DTI data for head movement and eddy current distortions with the non-diffusion-weighted imaging (the $\mathrm{B} 0$ image) as a reference. We fitted diffusion tensor models independently for each voxel and computed MD and FA maps. For the normalization of DTI, we first segmented the high-resolution T1-weighted images into gray matter, WM, and CSF by using the FMRIB Integrated Registration and Segmentation Tool (FIRST; http:// fsl.fmrib.ox.ac.uk/fsl/fslwiki/FIRST) and spatially normalized these images to the Montreal Neurological Institute coordinate space. By concatenating the transformation from the $\mathrm{B} 0$ image to the $\mathrm{T} 1$ image with the transformation from the $\mathrm{T} 1$ image to the Montreal Neurological Institute space (by using a nonlinear transformation), we obtained normalized FA and MD maps.

\section{GAMMA}

GAMMA is a machine-learning method that detects biomarkers from high-dimensional neuroimaging data. GAMMA has 2 important features: First, it has an embedded dimension-reduction mechanism. For high-dimensional neuroimaging data, the large number of candidate models turns the model inference into an underdetermined computational problem. Dimension reduction can ameliorate this problem. Second, GAMMA can detect multivariate interactions among brain regions characterizing a disorder by considering conditional independence among variables. To date, few neuroimaging data analysis methods have these 2 features.

The goal of GAMMA is to detect a set of brain regions that are jointly predictive of a group-membership variable $g$. Toward this end, GAMMA iteratively performs Markov Blanket identification 
and voxel-space partitioning. In Markov Blanket identification, GAMMA uses Bayesian methods to search for a set of voxels (called representative voxels) that are jointly predictive of $g$. The interactions among representative voxels and $g$ are described by a Bayesian network model. In voxel-space partitioning, for each representative voxel, GAMMA identifies voxels that are probabilistically equivalent to this representative voxel — that is, GAMMA searches for additional voxels that have similar associations with g. In this manner, each representative voxel is associated with a region of interest consisting of a representative voxel and its equivalent voxels. The output of GAMMA is a label field and a Bayesian network model. The label field includes a set of brain regions that are jointly most predictive of $g$, and each brain region in the label field is a ROI. The interactions among these brain regions and $g$ are described by the Bayesian network model. Details of the GAMMA algorithm are presented in Chen and Herskovits. $^{20}$

We calculated voxelwise mean and SD values for FA maps and compared each subject's FA map with the mean FA map. Decreased FA in WM has been consistently reported in patients with $\mathrm{MHE}^{12,14,15}$; therefore, we used a voxelwise threshold that is at multiple SDs below the mean value to threshold the FA map, resulting in a binary FA map, in which 1 represents an abnormal FA value and zero is normal. To reduce noise, we included only WM voxels with decreased FA value (determined by uncorrected $P<.05$ in a 2 -sample $t$ test). ${ }^{29}$ To ensure model stability, we compared 5 different thresholds: 0 SD, 0.25 SD, 0.50 SD, 0.75 SD, and 1.0 SD below the mean.

We performed similar preprocessing steps to generate binary MD maps. Because previous reports have consistently reported that patients with MHE have increased MD in $\mathrm{WM},{ }^{8,12,14-17}$ we set the voxelwise thresholds of the MD value at $0 \mathrm{SD}, 0.25 \mathrm{SD}, 0.50$ SD, $0.75 \mathrm{SD}$, and 1.0 SD above the mean.

After identifying FA and MD regions of interest that characterize group differences, we used the regional state inference algorithm ${ }^{20}$ to infer the state (normal/abnormal) for a particular ROI. Then we built Naive-Bayes classifiers (10-fold cross-validation) to discriminate patients with MHE and NHE on the basis of these regional state variables. The structure of the NaiveBayes classifier was [group membership $\rightarrow$ (biomarker $1, \ldots$, biomarker $\mathrm{m})$ ].

\section{Validation of Detected Biomarkers}

We performed 2 experiments to validate the biomarkers detected by GAMMA. For each subject, we extracted the average FA value for each ROI generated by GAMMA; let $F A\left(R O I_{\mathrm{k}}\right) / M D\left(R O I_{\mathrm{k}}\right)$ denote the average FA/MD value for the $k^{\text {th }}$ ROI.

First, we used Pearson correlation analysis to examine the relationship between $\mathrm{MD}\left(\mathrm{ROI}_{k}\right)$ or $\mathrm{FA}\left(\mathrm{ROI}_{k}\right)$ and the neurologic test score and the Child-Pugh score. Second, we performed survival analysis by using the detected biomarkers. We defined the date of the first DTI examination as day 1 , and each patient was followed up every 6 months. Fifty-five of 65 patients with cirrhosis completed follow-up studies until death or the end of the observation period (November 2012). We performed multivariate Cox regression (forward LR method) analysis to assess the associations of $\mathrm{MD}\left(\mathrm{ROI}_{k}\right)$ or $\mathrm{FA}\left(\mathrm{ROI}_{k}\right)$ with survival.

\section{RESULTS}

\section{Neurologic Assessments and Altered DTI Metrics}

Patients with MHE showed significantly impaired performance in all neurocognitive tests, compared with those with NHE (Table 1). Relative to patients with NHE, those with MHE showed increased mean MD values throughout the WM globally [NHE: $(0.805 \pm 0.020) \times 10^{-3} \mathrm{~mm}^{2} / \mathrm{s}$; MHE: $(0.821 \pm 0.035) \times 10^{-3}$ $\left.\mathrm{mm}^{2} / \mathrm{s} ; P=.025\right]$. Patients with MHE also had reduced mean FA values throughout the WM (NHE: $0.344 \pm 0.014$; MHE: $0.331 \pm$ 0.016; $P<.001$ ). The brain areas with increased MD values in MHE were the bilateral frontal lobes and the corpus callosum, and the areas with decreased FA values included the bilateral frontal, parietal, occipital, and temporal lobes; internal capsule; and corpus callosum (see the Appendix and On-line Figure). Moreover, in the MHE group, there was no area showing decreased MD or increased FA.

Additionally, we performed a post hoc power analysis based on FA. The observed effect size was 0.87 , which was considered large by using Cohen criteria. Power analysis with $\alpha=.05 \mathrm{dem}-$ onstrated that this study had power $=0.96$.

\section{Results of GAMMA Analysis}

Across different abnormality thresholds for the MD and FA maps, GAMMA consistently identified 2 spatially distributed WM regions that were jointly significantly predictive of the group-membership variable (Figs 1 and 2). Two ROIs identified in MD maps, denoted by $\mathrm{ROI}_{1}{ }^{\mathrm{MD}}$ and $\mathrm{ROI}_{2}{ }^{\mathrm{MD}}$, were predominant in the bilateral frontal lobes, precuneus, and the corpus callosum (Fig 1). Similarly, the 2 ROIs identified in FA maps, denoted by $\mathrm{ROI}_{1}{ }^{\mathrm{FA}}$ and $\mathrm{ROI}_{2}{ }^{\mathrm{FA}}$, included bilateral frontal lobes, the corpus callosum, and the left paracentral lobule (Fig 2). Of note, the ROIs that GAMMA identified from MD and FA maps largely overlapped.

\section{Results of Classification Analysis}

Table 2 shows classification performance based on these ROIs. Using MD maps, GAMMA achieved good classification accuracy (mean, 79.1\%; range, 75.4\%-81.5\%) across 5 distinct thresholds. The optimal classification performance was accuracy $=80.0 \%$, sensitivity $=75.9 \%$, and specificity $=83.3 \%$, with the threshold of 0.25 SD above the mean MD value. Using FA maps, GAMMA yielded higher classification accuracy (mean, 87.1\%; range, $83.1 \%-92.3 \%$ ) across the different thresholds. The optimal classification result was accuracy $=92.3 \%$, sensitivity $=100 \%$, and specificity $=86.1 \%$, which was obtained at the threshold of 0.75 SD below the mean FA value. The thresholds resulting in best classification result (eg, a threshold of 0.25 SD above the mean MD value or a threshold of 0.75 SD below mean FA value) were chosen and included in the following validation analyses.

\section{Results of Validation Analysis}

Table 3 shows that MD values of WM ROIs $\left[\mathrm{MD}\left(\mathrm{ROI}_{1}\right)\right.$ and $\mathrm{MD}\left(\mathrm{ROI}_{2}\right)$ ] were significantly positively correlated with the 


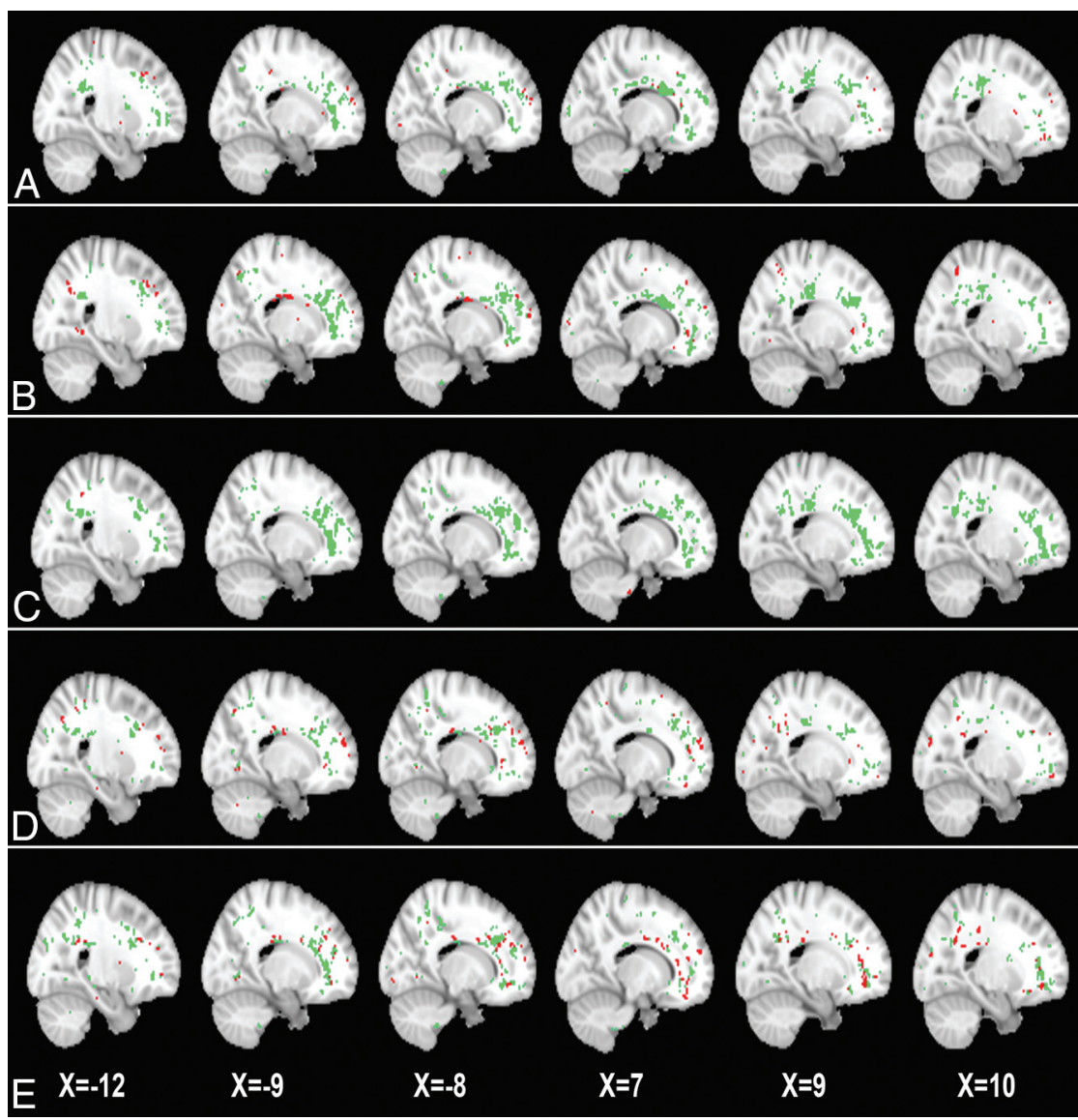

FIG 1. Two white matter ROIs generated by GAMMA, which characterize group differences on the MD map on the basis of voxelwise thresholds of the following: 0 SD (A), 0.25 SD (B), 0.50 SD (C), $0.75 \mathrm{SD}(D)$, and 1.0 SD $(E)$ above the mean voxelwise MD value. Green and red indicate the 2 ROls generated by GAMMA.

result of the Number Connection Test A and negatively correlated with the results of the Digit Symbol Test and Block Design Test. In contrast, FA values of WM ROIs $\left[\mathrm{FA}\left(\mathrm{ROI}_{1}\right)\right.$ and $\left.\mathrm{FA}\left(\mathrm{ROI}_{2}\right)\right]$ were significantly negatively correlated with the Number Connection Test A and positively correlated with the Digit Symbol Test and the Block Design Test scores. Longer time to complete Number Connection Test A and lower scores in the Digit Symbol Test and Block Design Test indicated poorer performance. Patients' Child-Pugh scores were significantly correlated with $\mathrm{MD}\left(\mathrm{ROI}_{1}\right), \mathrm{MD}\left(\mathrm{ROI}_{2}\right), \mathrm{FA}\left(\mathrm{ROI}_{1}\right)$, and $\mathrm{FA}\left(\mathrm{ROI}_{2}\right)$.

For survival analysis, the median follow-up period was 18.0 months. By the end of the observation, 15 patients (11 with MHE and 4 with NHE) had died from liver-related complications. Given the consensus that the Child-Pugh score is a variable with prognostic value for survival among patients with cirrhosis, ${ }^{2,25,26,28}$ we assessed the predictive values of MD and FA alterations by multivariate Cox regression, taking into account the Child-Pugh score as a covariate. Cox regression showed that $\mathrm{MD}\left(\mathrm{ROI}_{1}\right)$ (hazard ratio $[\mathrm{HR}]=4.253 ; 95 \% \mathrm{CI}, 1.218-$ $14.848 ; P=.023)$ was a predictor of survival, independent of the Child-Pugh score. Similarly, $\mathrm{MD}\left(\mathrm{ROI}_{2}\right)(\mathrm{HR}=3.948 ; 95 \% \mathrm{CI}$, $1.655-9.420 ; P=.002), \mathrm{FA}\left(\mathrm{ROI}_{1}\right)(\mathrm{HR}=0.823 ; 95 \% \mathrm{CI}, 0.696-$ $0.973 ; P=.022)$, and $\mathrm{FA}\left(\mathrm{ROI}_{2}\right)(\mathrm{HR}=0.805 ; 95 \% \mathrm{CI}, 0.671-0.965$;
$P=.019)$ showed prognostic value on survival as well, in addition to the ChildPugh assessment.

\section{DISCUSSION}

In this study, by using machine learning, we found that MD values in the bilateral frontal lobes, the precuneus, and the corpus callosum differentiate subjects with MHE and NHE; similarly, FA values in the bilateral frontal lobes, the corpus callosum, and the left paracentral lobule distinguish subjects with MHE from those with NHE. Meanwhile, MD and FA values of WM ROIs had prognostic value for survival, independent of the Child-Pugh score. These findings suggest the utility of measuring WM water molecular diffusivity for MHE detection.

WM edema (reflected by increased $M D)^{7-11}$ and impaired cerebral structures $^{12-14}$ have been demonstrated to play an important role in the MHE mechanisms. WM edema, which could be extracellular and/or cytotoxic in ori$\operatorname{gin},{ }^{8-10}$ is considered closely associated with a metabolic disorder of ammonia in the brain tissue of patients with MHE..$^{7,8,10,30}$ Although the neuropathologic mechanism of decreased FA is not well-understood in the setting of cirrhosis, one possible explanation is demyelination. Osmotic demyelination (due to inappropriate correction of hyponatremia ${ }^{31}$ ) has been noted in cirrhosis. ${ }^{32}$ Moreover, central nervous system inflammation has been shown to play an important role in $\mathrm{HE},{ }^{33}$ which may contribute to inflammatory demyelination as observed in other neurologic diseases such as multiple sclerosis. ${ }^{34}$ In addition, it has been found that factors released by astrocytes contribute to brain myelination, ${ }^{35}$ which would be impaired due to astrocytic dysfunction resulting from Alzheimer type II changes. ${ }^{7,8,10}$ Thus, reduction of WM FA may indicate impaired microstructural integrity in MHE.

Indeed, increased MD and decreased FA are also associated with the disruption of brain intrinsic networks, ${ }^{17,18}$ which can induce neurologic dysfunction in patients with MHE. In our study, the correlations between altered MD and FA values and psychometric results further suggest that WM abnormalities, especially in the corpus callosum and frontal and parietal lobes, are responsible for neurologic deficits such as psychomotor speed (reflected by the Number Connection Test A), attention and visual memory (reflected by the Digit Symbol Test), and visuospatial motor function (reflected by the Block Design Test).

We identified 2 spatially distributed WM ROIs, predominantly located in the bilateral frontal lobes, the corpus callosum, and bilateral parietal lobe, in MD maps as predictive of MHE. The ROIs identified from FA maps largely overlap those obtained 


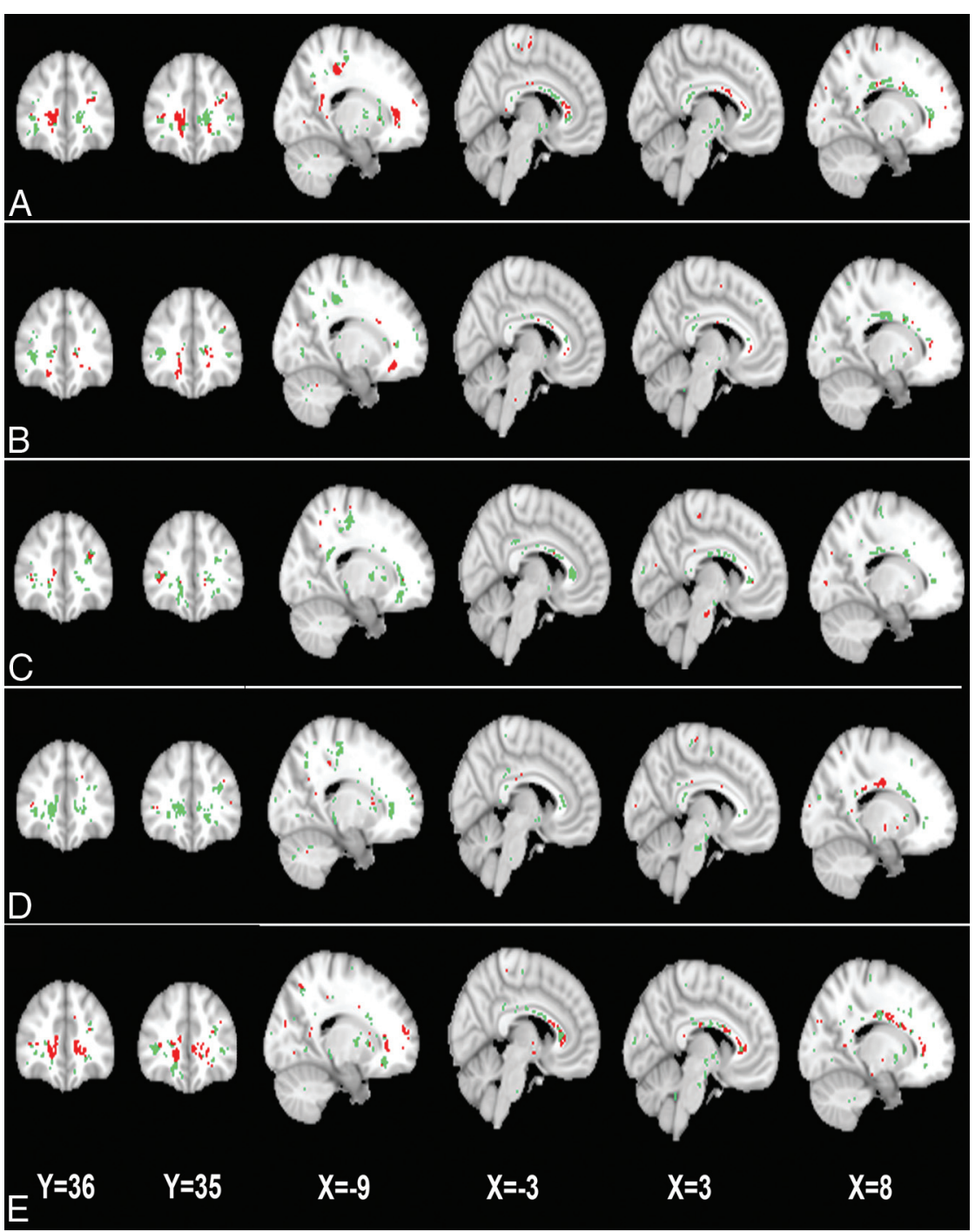

FIG 2. The 2 white matter ROIs generated by GAMMA, which characterize group differences in the FA on the basis of voxelwise thresholds of the following: 0 SD (A), $0.25 \mathrm{SD}(B), 0.50 \mathrm{SD}(C), 0.75$ $\mathrm{SD}(D)$, and 1.0 SD $(E)$ below the mean voxelwise FA value. Green and red indicate the 2 ROIs.

Table 2: Classification accuracy between patients with MHE and NHE using a single Bayesian network

\begin{tabular}{lccc}
\hline $\begin{array}{c}\text { Voxelwise Threshold and } \\
\text { Classification Network }\end{array}$ & Accuracy & Sensitivity & Specificity \\
\hline MD alone & & & \\
O SD above mean value & $78.5 \%$ & $75.9 \%$ & $80.6 \%$ \\
0.25 SD above mean value & $80.0 \%$ & $75.9 \%$ & $83.3 \%$ \\
0.50 SD above mean value & $75.4 \%$ & $58.6 \%$ & $88.9 \%$ \\
0.75 SD above mean value & $81.5 \%$ & $65.5 \%$ & $94.4 \%$ \\
1.0 SD above mean value & $80.0 \%$ & $62.1 \%$ & $94.4 \%$ \\
FA alone & & & \\
O SD below mean value & $84.6 \%$ & $75.9 \%$ & $91.7 \%$ \\
0.25 SD below mean value & $83.1 \%$ & $79.3 \%$ & $86.1 \%$ \\
0.50 SD below mean value & $89.2 \%$ & $86.2 \%$ & $91.7 \%$ \\
0.75 SD below mean value & $92.3 \%$ & $100 \%$ & $86.1 \%$ \\
1.0 SD below mean value & $86.2 \%$ & $72.4 \%$ & $97.2 \%$ \\
\hline
\end{tabular}

from MD maps; this overlap indicates that these WM regions are predominantly affected by MHE pathology. Increased ammonia levels have been found to be associated with the increased ADC in the frontal and parietal WM. ${ }^{16}$ In addition, positron-emission tomography studies have indicated that an altered glial cell state, an important cause of MHE, occurs in the frontal WM and in the corpus callosum. ${ }^{36}$ The frontal and parietal WM and corpus callosum (particularly the anterior part) are the regions most commonly reported to demonstrate increased water content ${ }^{11}$ or diffusivity ${ }^{9,10,12,15,16}$ and decreased FA. ${ }^{15}$ When patients with cirrhosis in the intermediate state are excluded, Sugimoto et $\mathrm{al}^{16}$ reported $70 \%-90 \%$ sensitivity and $85 \%-90 \%$ specificity in classification of MHE and NHE, based on a frontal or parietal WM ADC metric. Their results indicate, to some extent, the usefulness of measuring WM diffusion features in MHE detection and are in line with our findings.

Increased regional MD has been found to be predictive of the development of overt $\mathrm{HE},{ }^{16}$ which has an ominous prognostic value on survival. ${ }^{2}$ Meanwhile, MHE is associated with increased risk of an episode of overt HE. ${ }^{5}$ It is believed that MHE (measured by neuropsychological tests) has prognostic value on survival in patients with cirrhosis with covert HE. ${ }^{25}$ These progressive patterns of disease may explain why we found increased MD to be predictive of survival for patients with cirrhosis with covert HE. Brain edema has been reported to be fatal, especially in highgrade $\mathrm{HE}$, due to increased intracranial pressure. $^{37,38}$ Moreover, our findings regarding the predictive values of $\mathrm{MD}$ and FA on survival agree with the consensus that brain edema ${ }^{9-11}$ and structural impairment ${ }^{12,13}$ progress with advanced disease.

One strength of this study is the application of an advanced machine-learning method, GAMMA, to the detection of MHErelated biomarkers. ${ }^{20-22}$ GAMMA is a nonparametric and multivariate Bayesian approach to model complex nonlinear multivariate associations among image features and the clinical variables. GAMMA automatically generates ROIs that can be used to differentiate MHE from NHE; this feature distinguishes our study from previous DWI studies, which relied on prespecified ROIs. ${ }^{8-10,16}$ The consistency of classification across distinct thresholds suggests the validity of these results. Moreover, the significance of MD ROIs and FA ROIs in predicting survival provides further evidence regarding the validity of biomarkers detected by GAMMA.

One limitation of our study is that we confined our analysis to WM regions, where brain edema predominantly occurs in $\mathrm{MHE}^{11,15}$; however, several studies have indicated that other cerebral regions, such as subcortical nuclei, ${ }^{9,10}$ may also manifest 
Table 3: Pearson correlation coefficients of MD and FA values with neurologic performances and Child-Pugh score among patients with cirrhosis and covert hepatic encephalopathy

\begin{tabular}{lrrrrr}
\hline & \multicolumn{2}{c}{ MD Map } & & \multicolumn{2}{c}{ FA Map } \\
\cline { 2 - 3 } \cline { 5 - 6 } \multicolumn{1}{c}{ Variables } & ROI1 & ROI2 & & ROI1 & ROI2 \\
\hline Number Connection Test A & $0.38^{\mathrm{a}}$ & $0.41^{\mathrm{a}}$ & & $-0.59^{\mathrm{a}}$ & $-0.63^{\mathrm{a}}$ \\
Digit Symbol Test & $-0.51^{\mathrm{a}}$ & $-0.52^{\mathrm{a}}$ & & $0.71^{\mathrm{a}}$ & $0.68^{\mathrm{a}}$ \\
Block Design Test & $-0.32^{\mathrm{a}}$ & $-0.37^{\mathrm{a}}$ & & $0.51^{\mathrm{a}}$ & $0.56^{\mathrm{a}}$ \\
Child-Pugh score & $0.33^{\mathrm{a}}$ & $0.37^{\mathrm{a}}$ & & $-0.27^{\mathrm{b}}$ & $-0.34^{\mathrm{a}}$ \\
\hline
\end{tabular}

${ }^{\mathrm{a}} P<.01$.

${ }^{\mathrm{b}} \mathrm{P}<.05$.

low-grade edema. The second limitation is that we used a suboptimal DTI sequence with 6 diffusion-encoding gradient directions, due to the finite capability of our MR imaging scanner. The third limitation is that we only used DTI to generate biomarkers for MHE. Other MR imaging models (eg, resting-state $\mathrm{AMRI}^{39}$ ) have been reported to be useful in distinguishing MHE from NHE as well. DTI may be more readily applicable in the clinical setting because it is more convenient to obtain DTI relative to restingstate fMRI. However, the combination of these MR imaging techniques may yield more accurate classification and is an area for future extension of this research.

\section{CONCLUSIONS}

Combining advanced machine-learning techniques and DTI can generate biomarkers that identify patients with MHE among patients with cirrhosis with covert hepatic encephalopathy; both increased MD and decreased FA are predictive of survival. Our findings may be helpful in detecting MHE and improving subsequent treatment.

\section{APPENDIX}

\section{Between-Group Comparison}

The 2-sample $t$ tests were performed to determine the regions with an MD/FA difference between 2 groups in a voxelwise way. The statistical threshold was set at $P<.05$ (the combination of $P<.005$ for single voxel and a minimum cluster size of 120 voxels), which was determined by Monte Carlo simulations by using the AlphaSim program (http://afni.nimh.nih.gov/afni).

Disclosures: Edward H. Herskovits—UNRELATED: Expert Testimony: Medicolegal; Grants/Grants Pending: Microsoft,* State of Maryland.* *Money paid to the institution.

\section{REFERENCES}

1. Ferenci P, Lockwood A, Mullen K, et al. Hepatic encephalopathy: definition, nomenclature, diagnosis, and quantification-final report of the working party at the 11th World Congresses of Gastroenterology, Vienna, 1998. Hepatology 2002;35:716-21

2. Stewart CA, Malinchoc M, Kim WR, et al. Hepatic encephalopathy as a predictor of survival in patients with end-stage liver disease. Liver Transpl 2007;13:1366-71

3. Bajaj JS, Wade JB, Sanyal AJ. Spectrum of neurocognitive impairment in cirrhosis: implications for the assessment of hepatic encephalopathy. Hepatology 2009;50:2014-21

4. Groeneweg M, Quero JC, De Bruijn I, et al. Subclinical hepatic encephalopathy impairs daily functioning. Hepatology 1998;28:45-49

5. Romero-Gómez M, Boza F, Garcia-Valdecasas MS, et al. Subclinical hepatic encephalopathy predicts the development of overt hepatic encephalopathy. Am J Gastroenterol 2001;96:2718-23

6. Prasad S, Dhiman RK, Duseja A, et al. Lactulose improves cognitive functions and health-related quality of life in patients with cirrhosis who have minimal hepatic encephalopathy. Hepatology 2007; 45:549-59

7. Córdoba J, Alonso J, Rovira A, et al. The development of low-grade cerebral edema in cirrhosis is supported by the evolution of (1) Hmagnetic resonance abnormalities after liver transplantation. J Hepatol 2001;35:598-604

8. Chavarria L, Alonso J, Garcia-Martinez R, et al. Biexponential analysis of diffusion-tensor imaging of the brain in patients with cirrhosis before and after liver transplantation. AJNR Am J Neuroradiol 2011;32:1510-17

9. Kale RA, Gupta RK, Saraswat VA, et al. Demonstration of interstitial cerebral edema with diffusion tensor MR imaging in type $\mathrm{C}$ hepatic encephalopathy. Hepatology 2006;43:698-706

10. Lodi R, Tonon C, Stracciari A, et al. Diffusion MRI shows increased water apparent diffusion coefficient in the brains of cirrhotics. Neurology 2004;62:762-66

11. Shah NJ, Neeb H, Kircheis G, et al. Quantitative cerebral water content mapping in hepatic encephalopathy. Neuroimage 2008;41: 706-17

12. Chen HJ, Wang Y, Zhu XQ, et al. White matter abnormalities correlate with neurocognitive performance in patients with $\mathrm{HBV}$-related cirrhosis. J Neurol Sci 2012;321:65-72

13. Guevara M, Baccaro ME, Gomez-Anson B, et al. Cerebral magnetic resonance imaging reveals marked abnormalities of brain tissue density in patients with cirrhosis without overt hepatic encephalopathy. J Hepatol 2011;55:564-73

14. Qi R, Zhang LJ, Zhong J, et al. Grey and white matter abnormalities in minimal hepatic encephalopathy: a study combining voxelbased morphometry and tract-based spatial statistics. Eur Radiol 2013;23:3370-78

15. Kumar R, Gupta RK, Elderkin-Thompson V, et al. Voxel-based diffusion tensor magnetic resonance imaging evaluation of low-grade hepatic encephalopathy. J Magn Reson Imaging 2008;27:1061-68

16. Sugimoto R, Iwasa M, Maeda M, et al. Value of the apparent diffusion coefficient for quantification of low-grade hepatic encephalopathy. Am J Gastroenterol 2008;103:1413-20

17. Qi R, Xu Q, Zhang LJ, et al. Structural and functional abnormalities of default mode network in minimal hepatic encephalopathy: a study combining DTI and fMRI. PLoS One 2012;7:e41376

18. Lin WC, Hsu TW, Chen CL, et al. Connectivity of default-mode network is associated with cerebral edema in hepatic encephalopathy. PLoS One 2012;7:e36986

19. Bajaj JS, Saeian K, Verber MD, et al. Inhibitory control test is a simple method to diagnose minimal hepatic encephalopathy and predict development of overt hepatic encephalopathy. Am J Gastroenterol 2007;102:754-60

20. Chen R, Herskovits EH. Graphical model based multivariate analysis (GAMMA): an open-source, cross-platform neuroimaging data analysis software package. Neuroinformatics 2012;10:119-27

21. Chen R, Herskovits EH. Graphical-model-based morphometric analysis. IEEE Trans Med Imaging 2005;24:1237-48

22. Chen R, Pawlak MA, Flynn TB, et al. Brain morphometry and intelligence quotient measurements in children with sickle cell disease. $J$ Dev Behav Pediatr 2009;30:509-17

23. Chen R, Herskovits EH. Graphical-model-based multivariate analysis of functional magnetic-resonance data. Neuroimage 2007;35:635-47

24. Mueller SG, Young K, Hartig M, et al. A two-level multimodality imaging Bayesian network approach for classification of partial epilepsy: preliminary data. Neuroimage 2013;71:224-32

25. Dhiman RK, Kurmi R, Thumburu KK, et al. Diagnosis and prognostic significance of minimal hepatic encephalopathy in patients with cirrhosis of liver. Dig Dis Sci 2010;55:2381-90

26. Amodio P, Del Piccolo F, Marchetti P, et al. Clinical features and survival of cirrhotic patients with subclinical cognitive alterations 
detected by the number connection test and computerized psychometric tests. Hepatology 1999;29:1662-67

27. Bajaj JS, Hafeezullah M, Franco J, et al. Inhibitory control test for the diagnosis of minimal hepatic encephalopathy. Gastroenterology 2008;135:1591-600.e1

28. Romero-Gómez M, Grande L, Camacho I. Prognostic value of altered oral glutamine challenge in patients with minimal hepatic encephalopathy. Hepatology 2004;39:939-43

29. Bourgon R, Gentleman R, Huber W. Independent filtering increases detection power for high-throughput experiments. Proc Natl Acad Sci U S A 2010;107:9546-51

30. Mardini $\mathrm{H}$, Smith FE, Record CO, et al. Magnetic resonance quantification of water and metabolites in the brain of cirrhotics following induced hyperammonaemia. J Hepatol 2011;54:1154-60

31. Restuccia T, Gomez-Anson B, Guevara M, et al. Effects of dilutional hyponatremia on brain organic osmolytes and water content in patients with cirrhosis. Hepatology 2004;39:1613-22

32. Yu C, Sharma N, Saab S. Hyponatremia: clinical associations, prognosis, and treatment in cirrhosis. Exp Clin Transplant 2013;11:3-11

33. Rodrigo R, Cauli O, Gomez-Pinedo U, et al. Hyperammonemia induces neuroinflammation that contributes to cognitive impair- ment in rats with hepatic encephalopathy. Gastroenterology 2010; 139:675-84

34. Zhornitsky S, Wee Yong V, Koch MW, et al. Quetiapine fumarate for the treatment of multiple sclerosis: focus on myelin repair. CNS Neurosci Ther 2013;19:737-44

35. Ishibashi T, Dakin KA, Stevens B, et al. Astrocytes promote myelination in response to electrical impulses. Neuron 2006;49: $823-32$

36. Cagnin A, Taylor-Robinson SD, Forton DM, et al. In vivo imaging of cerebral "peripheral benzodiazepine binding sites" in patients with hepatic encephalopathy. Gut 2006;55:547-53

37. Donovan JP, Schafer DF, Shaw BW Jr, et al. Cerebral oedema and increased intracranial pressure in chronic liver disease. Lancet 1998;351:719-21

38. Joshi D, O'Grady J, Patel A, et al. Cerebral oedema is rare in acuteon-chronic liver failure patients presenting with high-grade hepatic encephalopathy. Liver Int 2014;34:362-66

39. Chen HJ, Wang Y, Zhu XQ, et al. Classification of cirrhotic patients with or without minimal hepatic encephalopathy and healthy subjects using resting-state attention-related network analysis. PLOS One 2014;9:e89684 